\title{
A BISCRITUALIDADE NOS MAIS ANTIGOS MANUAIS DA LÍNGUA ALEMÃ PARA UM PÚBLICO PORTUGUÊS
}

\section{BISCRIPTALITY IN THE OLDEST GERMAN HANDBOOKS FOR PORTUGUESE READERS}

\author{
Rolf Kemmler \\ Universidade de Trás-os-Montes e Alto Douro \\ Centro de Estudos em Letras \\ kemmler@utad.pt
}

\section{RESUMO:}

Com o fim de servir como ponto de partida para futuros estudos que visam analisar o uso da Fraktur (os chamados 'carateres góticos') ou mesmo das vertentes manuscritas Kurrentschrift vs. Sütterlinschrift nos manuais da língua alemã para um público português, é com base no artigo do investigador austríaco Bernd Marizzi (2012b) sobre os inícios da tradição espanhola e a biscritualidade observada nas suas manifestações, que o presente artigo visa oferecer algumas reflexões sobre a ocorrência semelhante de formas de escrita divergentes na representação das línguas alemã e portuguesa em obras metalinguísticas que têm o português como metalinguagem.

PALAVRAS-CHAVE: Biscritualidade; língua alemã, sistemas de escrita; carateres góticos, Fraktur, Kurrent; Sütterlin; Antiqua.

\begin{abstract}
:
In order to serve as a starting point for future studies that aim to analyze the use of the Fraktur (the so-called 'gothic characters') or even the handwritten scripts Kurrentschrift vs. Sütterlinschrift in the German language manuals for a Portuguese public, based on an article of the Austrian researcher Bernd Marizzi (2012b) dedicated to the beginnings of the Spanish tradition and the biscriptality that can be observed in its manifestations, the present article aims to offer some reflections on the similar occurrence of divergent forms of writing in the representation of the German and Portuguese languages in metalinguistic works that have the Portuguese as metalanguage.
\end{abstract}

KEYWORDS: Biscriptality; German language; writing systems; Gothic characters; Fraktur; Kurrent; Sütterlin; Antiqua 


\section{Introdução}

No último capítulo do seu artigo sobre a Gramatica de la lengua alemana: dividida en tres partes do dominicano espanhol Antonio de Villa (fl. 1788-1811), o investigador austríaco Bernd Marizzi (2012a) dedica-se detidamente a um tema muito importante aos primórdios da aprendizagem e do ensino do alemão como língua estrangeira $(\mathrm{DaF})$, nomeadamente à questão da 'biscritualidade" ('Zweischriftigkeit'; inglês 'biscriptality') do alemão (no sentido de Glück 1994: 746 ou Bunčić 2011; 2016: 36) e à reprodução resultante dos tipos usuais em (quase) todas as publicações de língua alemã:

Es conocido que en el ámbito cultural germano se utilizaba hasta hace no mucho tiempo (1941) cierto tipo de letra, distinta a la antigua letra latina redonda (antiqua): la letra gótica o fracturada (Marizzi 2012a: 141)

Como Bernd Marizzi afirma ainda, a questão do tipo de letra de impressão (e das variantes associadas com a letra manuscrita, de que os estudantes da língua alemã da época não poderiam prescindir) não é, portanto, pouco problemática para o contexto da impressão de manuais didáticos do alemão como língua estrangeira, publicados fora da Alemanha ou da Europa Central:

En el siglo XVIII, cuando Antonio de Villa quiso mandar imprimir su gramática se encontró con que en España ya no había ni tipos alemanes ni "fundición de caracteres Alemanes (...) ni impresor dispuesto a costearlo" (Consejos 11.279, exp. 2, Enero de 1791). Por este motivo se vio obligado a imprimir la gramática "en el carácter del Reyno, sustituyendo la letra redonda por la Alemana, y la bastardilla ó cursiva por la Española, ó para explicar la Lengua Alemana" (Villa 1792: 2) (Marizzi 2012a: 141).

Embora possa ter havido autores de obras metalinguísticas que prescindiram do tipo 'gótico' alemão (Fraktur) por motivos didáticos - tal como Corvo Sánchez (2002: 256; ver também Corvo Sánchez 2007, 2011) conseguiu comprovar para o caso do Thesaurvs fundamentalis quinqve lingvarvm de Zumaran (1626, I: [XII]) - parece óbvio que só à primeira vista o problema 'puramente tipográfico' da não-existência de tipos de Fraktur, ou a correspondente falta

No seu artigo em língua espanhola, Marizzi (2012a: 142) coerentemente introduz o neologismo 'biscriptualidad', até então desconhecido em espanhol, ao qual em língua portuguesa corresponderá o termo até agora semelhantemente desconhecido de 'biscritualidade'. 
de experiência em lidar com este tipo, não só na Madrid de finais do século XVIII terá constituído um obstáculo para a edição de produtos editoriais em língua alemã, mas provavelmente também no Portugal vizinho de finais do século XVIII ou inícios do século XIX. Também é questionável se mais tarde todas as tipografias estavam munidas dos tipos de fratura, ou se, no caso dos manuais de língua alemã publicados por autores residentes em Portugal para o mercado Português, ${ }^{2}$ a escolha de um local de impressão estrangeiro poderia ser relacionada com este problema de natureza principalmente tipográfica.

\section{O uso das escritas 'Fraktur', 'Kurrent' und 'Sütterlin'}

O tipo geralmente referido na cultura alemã como 'Fraktur' desenvolveu-se no século XVI, «[...] um und nach der Jahrhundertmitte als hochkalligraphische Buchschrift [...]» (Schneider 2014: 80)»3 (Schneider 2014: 80) como resultado dos 'tipos góticos' (Overgaauw 1994; Schneider 2014: 28-86) desenvolvidos com base na tradição manuscrita amplamente difundida na Europa em forma da minúscula carolíngia, devendo, enfim, a sua existência ao seu uso como escrita dos documentos da secretaria do imperador Maximiliano I (1459-1519, a partir de 1508 imperador do Sacro Império Romano ) (veja-se Šedivý 2007 com mais informações). Do ponto de vista paleográfico, Schneider (2014: 80) carateriza a Fraktur como «[...] eine sehr stilisierte Bastarda mit den stark verlängerten, an- und abschwellenden, ,spindelförmigen ' $\mathrm{f}$ und langen $\mathrm{f}$, deutlichen Druckunterschieden, von rechts her oder mit Schleifen beginnenden Oberlängen und mit feinen Verzierungen $[\ldots]$... ${ }^{4}$, sendo os grafemas (ou melhor alógrafos de $<\mathrm{f}, \mathrm{s}>$ ) realizados em Fraktur como $<\mathrm{f}, \mathrm{p} .{ }^{5}$

2 Assim, por exemplo, Paris no caso das obras Nova grammatica theorica e prática para o estudo da lingua allemã (Apell ${ }^{1}$ 1897, ${ }^{2}$ 1898a), Selecta de Leituras Allemãs (Apell 1898b), bem como da Grammatica Allemã (Campos 1898a) e das Leituras allemãs (Campos 1898b), todas publicadas pela editora parisiense 'Aillaud \& C. ${ }^{\text {ia }}$ Casa Editora e de Commissão'.

$3 \quad[(\ldots)$ por volta de e depois da metade do século como da escrita impressa altamente caligráfica (...)]. Todas as traduções do alemão para o português são da nossa autoria, sendo colocadas em parênteses retos.

$4 \quad\left[(. .\right.$.$) uma Bastarda muito estilizada com os f 'fusiformes' e \int$ longos altamente alongados, crescentes e decrescentes, diferenças de impressão consideráveis, extremidades superiores a começar à direita ou com voltas e com ornamentos finos (...)].

5 Tendo em mente a morfologia dos carateres góticos, parece óbvio que particularmente a oposição gráfica $\langle\mathfrak{f}>\sim<\hat{f}>($ i.é $<\mathfrak{f}>\sim<\mathrm{s}>$ ) poderia ter sido especialmente difícil para um público de um manual de alemão quando este ainda não tivesse a competência suficiente na leitura do alemão. 
A forma de como o traçado tanto da escrita da 'Antiqua' latina (ie 'a antiga') como da 'Fraktur' de cunho germânico (substantivo deverbal do latim frangere, frangō, fractus) deve ser compreendido desde o ponto de vista gráfico ou tipográfico, é ilustrada graficamente por Plata (1999: 63) como se segue:
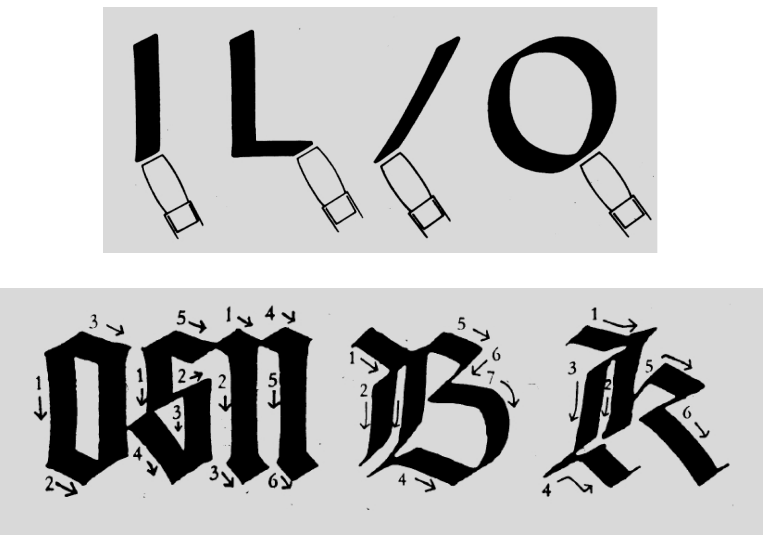

Uma vez que nos pareça mais provável que impressões incunábulas portuguesas, tais como as primeiras obras metalinguísticas impressas em Portugal de Pastrana / Rombo (1497), Rombo (1497) e Martins (1497) para o latim, e Oliveira (1536) para português, não sejam impressas em Fraktur, mas em 'letras góticas' não itálicas no sentido de uma 'textualis' (cf. Schneider 2014: 38-42), (isto é, sem os elementos de bastarda itálicos, que são tão caraterísticos da Fraktur de estilo alemão), o complexo desenvolvimento da escrita impressa na Europa da gótica rumo à Antiqua ao longo dos séculos é caraterizada pertinentemente em detalhe por Christina Killius como se segue:

$\mathrm{Zu}$ Beginn des 16. Jahrhunderts überwogen im Buchdruck noch die Schriften der gotischen Tradition. Allmählich begann sich die Antiqua durchzusetzen, die anfänglich aber lediglich für humanistische Texte zur Anwendung kam. Italien war schließlich das erste Land, das zu einem allgemeinen Gebrauch der Antiquatypen überging. Frankreich und Spanien folgten in der ersten Hälfte des 16. Jahrhunderts, während es in England erst um 1700 zu einer einheitlichen Verwendung der Antiqua kam. Die Niederlande und Schweden verwendeten die Antiqua als allgemeine Schrift dann im 18. Jahrhundert. Deutschland nahm in 
dieser typographischen Tradition eine Sonderrolle ein. Bis 1941, als durch einen Erlaß der nationalsozialistischen Machthaber die Antiqua zur Allgemeinschrift erhoben wurde, fand sie lediglich für den Druck fremdsprachiger und lateinischer Texte Verwendung (Killius 1999: 68). ${ }^{6}$

Embora a continuação do uso da Fraktur tivesse sido controversa na Alemanha desde finais do século XIX (até houve uma Allgemeiner Verein für Altschrift [Associação Geral para a Escrita Antiga] que tentou impor a Antiqua no Reich alemão a partir de 1880), o relacionamento entre as escritas do alemão nos tempos modernos geralmente se aplicava o que Süß (1995: 6) resume retrospetivamente de maneira simplista: «Die Fraktur als langsam schreibbare Buchschrift, die Kurrent (von lat.: currere = laufen) als Konzept- und Verkehrsschrift und die stilistisch dazwischenstehende Kanzlei als Aktenreinschrift» [A Fraktur como a escrita impressa lentamente gravável, a Kurrent (de lat: currere $=$ correr) como uma escrita de rascunho e de comunicação e a Kanzlei estilisticamente intermediária para a caligrafia de processos], uma realidade que Schneider (2014: 84-86) especifica desde o ponto de vista paleográfico:

Eine aus der Kanzleibastarda entwickelte, im frühen 16. Jahrhundert neu aufkommende Schriftart ist schließlich die Kurrentschrift. [...] Es ist eine rechtsschräge Schrift mit Schleifen an den Oberlängen und den stark verlängerten $\mathrm{f}$ - und f-Schäften der älteren Kanzleibastarden, aber mit zahlreichen kursiv in einem einzigen Federzug geschriebenen Formen wie g, $\mathrm{p}$ oder rundem $\mathrm{s}$; $\mathrm{h}$ und $\mathrm{z}$ haben durchgezogene Schleifen an den Unterbögen, a und $r$ werden in zwei mit kleinen Schleifen verbundene Bestandteile zerlegt, eine charakteristische Form hat das wie geknüpft wirkende e [...]. Diese Kurrentschrift ist die Grundlage

6 [No início do século XVI, na impressão de livros ainda predominavam os tipos da tradição gótica. Gradualmente, a Antiqua começou a prevalecer, que inicialmente, porém, apenas era usada para textos humanísticos. A Itália, afinal, foi o primeiro país a passar para um uso geral da antiqua. França e Espanha seguiram na primeira metade do século XVI, enquanto na Inglaterra um uso uniforme da antiqua somente se tenha verificado por volta de 1700 . A Holanda e a Suécia passaram a usar Antiqua como tipo geral desde o século XVIII. AAlemanha desempenhou um papel especial nessa tradição tipográfica. Até 1941, quando a antiqua foi elevada ao estatuto de tipo geral por um decreto dos governantes nacional-socialistas, ela somente foi usada para a impressão de textos em latim e em línguas estrangeiras modernas]. 
der späteren Schriften des 17.-19. Jahrhunderts für deutsche Texte, deren letzte Entwicklungsstufe noch bis zu ihrer Aufhebung 1941 in den deutschen Schulen als Grundschrift gelehrt wurde. ${ }^{7}$

Além disso, existe ainda outra escrita (desenvolvida e padronizada, enfim, a partir da variante oitocentista da Kurrentschrift) de inícios do século XX, que até hoje é provavelmente a escrita histórica alemã mais conhecida. Trata-se da 'Normschrift nach Ludwig Sütterlin' [escrita normal segundo Ludwig Sütterlin], 'Sütterlinschrift' [escrita de Sütterlin], muitas vezes só mencionada como 'Sütterlin': ${ }^{8}$

Ludwig Sütterlin (1865-1917), dessen Name oft fälschlich als Sammelbegriff für alle deutschen Schreibschriften verwendet wird, war der landläufig wohl bekannteste Erneuerer. Er stellte seine Buchstaben senkrecht, vereinfachte deren Formen auf ein Mindestmaß und legte die Lineatur auf das Verhältnis 1:1:1 fest. Seine Schulausgangsschrift [...] fand ab 1914 versuchsweise, ab 1924 verbindlich [...] in den preußischen Grundschulen Eingang und wurde bis 1930 bereits in den meisten deutschen Ländern im Unterricht verwendet [...] (Süß 1995: 7).

7 [Uma escrita desenvolvida a partir da bastarda de chancelaria, que emerge em inícios do século XVI, é finalmente a escrita corrente. (...) É uma escrita cursiva com laços nas extremidades superiores e com as hastes de $\mathrm{f}$ e $\mathrm{s}$ das antigas bastardas de chancelaria fortemente alongadas, mas com numerosas formas cursivas escritas numa única pluma, como g, p ou s redondo; h e z têm laços contínuos nos arcos inferiores, a e r são divididos em duas partes ligadas por pequenos laços, o e que parece ligado tem uma forma característica [...]. Esta escrita corrente é a base das escritas posteriores dos séculos XVIII-XIX para textos alemães, cujo último estágio de desenvolvimento ainda era ensinado nas escolas alemãs como escrita básica até sua revogação em 1941]. Também Seidl (1996: 9) observa o seguinte em relação à forma de letra da Kurrentschrift ao longo dos séculos: "Gegen Ende des 16. Jahrhunderts hat die Kurrentschrift im großen und ganzen ihre bis in das 20. Jahrhundert gültige Form erhalten. Von dieser Zeit an ändern sich die Formen der Buchstaben bis auf einige wenige Ausnahmen kaum mehr [...], es modifiziert sich lediglich der Duktus (Gesamteindruck) der Schrift» [Até finais do século XVI, a escrita Kurrent recebeu largamente a sua forma válida até o século XX. A partir desse momento, as formas dos carateres, com poucas exceções, dificilmente mudam [...], apenas muda o estilo (impressão geral) do tipo de letra].

8 [Ludwig Sütterlin (1865-1917), cujo nome muitas vezes erroneamente é usado como um termo coletivo para todas as escritas cursivas alemães, foi provavelmente o renovador mais conhecido do país. Ele colocou os seus carateres verticalmente, simplificou as suas formas para um mínimo e definiu a lineatura para a proporção de 1: 1:1. A sua caligrafia oficial (...) foi utilizada provisoriamente a partir de 1914 e obrigatoriamente a partir de 1924 (...) nas escolas primárias prussianas e já era usada por 1930 na maioria dos estados alemães (...)]. 
Ao tomarmos em consideração as caraterísticas das duas escritas impressas (Antiqua Fraktur) e o estilo cursivo da Kurrent em comparação com a Sütterlinschrift atualmente escrita, baseadas no alfabeto alemão estas escritas podem ser retratadas da seguinte maneira (fonte TrueType 'Times New Roman' em comparação com 'Leipzig Fraktur', 'Wiegel Kurrent', 'Sütterlin', veja-se também o resumo em Seidl 1996: 14):

\begin{tabular}{|c|c|c|c|c|c|c|c|c|c|c|c|c|c|c|c|c|c|c|c|c|c|c|c|c|c|c|}
\hline \multicolumn{5}{|c|}{$\begin{array}{l}\text { Kleinbuchstaben } \\
\text { letras minúsculas }\end{array}$} & \multicolumn{8}{|c|}{$\begin{array}{l}\text { Retembuchffaben } \\
\text { letraí mtmufculaf }\end{array}$} & \multicolumn{7}{|c|}{ 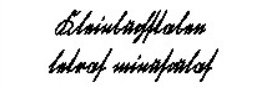 } & \multicolumn{7}{|c|}{ 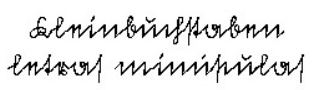 } \\
\hline Antiqua & $\mathrm{a}$ & b & $\mathrm{C}$ & d & e & $\mathrm{f}$ & $\mathrm{g}$ & $\mathrm{h}$ & i & J & $\mathrm{k}$ & 1 & $\mathrm{~m}$ & $\mathrm{n}$ & $\mathrm{O}$ & $\mathrm{p}$ & $\mathrm{q}$ & $\mathrm{r}$ & $S$ & l & $\mathrm{u}$ & $\mathrm{v}$ & $\mathrm{w}$ & $\mathrm{x}$ & $\mathrm{y}$ & z \\
\hline Fraktur & $\mathfrak{a}$ & b & c & $D$ & e & $f$ & $\mathfrak{g}$ & h) & $\mathfrak{t}$ & f & i & l & $\mathrm{m}$ & $\mathfrak{n}$ & D & $p$ & $q$ & $\mathfrak{r}$ & f & $t$ & $\mathfrak{u}$ & $\mathfrak{v}$ & wo & $x$ & b) & $z$ \\
\hline Kurrent & $\approx$ & 6 & f & 9 & $*$ & 1 & $g$ & 1 & 7 & $j$ & 6 & $\ell$ & $m$ & * & * & 9 & $q$ & * & l & $l$ & \# & $a$ & $*$ & 8 & 8 & 8 \\
\hline Sütte & or & b & i & 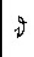 & $n$ & $f$ & $g$ & $f$ & $i$ & $j$ & 2. & $l$ & $m$ & $n$ & $\theta$ & p & $q$ & 伶 & 1 & 4 & $\breve{n}$ & 10 & no & $\varphi$ & 10 & $z$ \\
\hline \multicolumn{6}{|c|}{$\begin{array}{l}\text { Großbuchstaben } \\
\text { letras minúsculas }\end{array}$} & \multicolumn{7}{|c|}{$\begin{array}{l}\text { Srop̧buchftaben } \\
\text { letrai matuf culaf }\end{array}$} & \multicolumn{7}{|c|}{ Groptiofflotisen } & \multicolumn{7}{|c|}{ 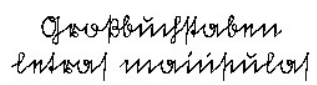 } \\
\hline Antiqua & A & B & C & D & $\mathrm{E}$ & $F$ & $\mathrm{G}$ & $\mathrm{H}$ & I & $\mathbf{J}$ & K & ( & $\mathbf{M}$ & $\mathrm{N}$ & $\mathrm{O}$ & $\mathrm{P}$ & $\mathrm{Q}$ & $\mathrm{R}$ & $S$ & $\mathrm{~T}$ & $\mathrm{U}$ & $\mathrm{V}$ & W & $\mathrm{x}$ & $\mathrm{Y}$ & $\mathrm{Z}$ \\
\hline Fraktur & |21 & 63 & (5) & $\mathfrak{D}$ & E & $\mathfrak{F}$ & 33 & H & $\mid \Im$ & 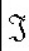 & $\Re$ & \& & $\mathfrak{M}$ & $\mathfrak{R}$ & $\mathfrak{D}$ & $P$ & $\mathfrak{Q}$ & $\pi$ & 5 & $\mathfrak{F}$ & ul & $\mathfrak{B}$ & $\mathfrak{W}$ & $\mathfrak{X}$ & Y) & 3 \\
\hline Kurrent & $a$ & & & 8 & $\notin$ & & $g$ & $\$$ & 2 & & 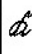 & $\mathscr{L}$ & 92 & $\mathscr{Z C}$ & 0 & & $q$ & $\not 2$ & $g$ & $y$ & $\mathcal{U}$ & $\mathscr{D}$ & $\mathscr{D}$ & $\mathscr{L}$ & $\not 2$ & g \\
\hline Sütterlin & a & $p_{p}$ & $\delta$ & $\vartheta$ & $\Phi$ & of & g & $b$ & 3 & 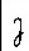 & de & $\mathscr{L}$ & 200 & $\gamma$ & $\theta$ & 2 & q & z & $\gamma$ & a & $u$ & 10 & 20 & $x$ & $2 y$ & $z$ \\
\hline
\end{tabular}

Para além disso, na escrita Fraktur, ainda são de considerar as ligaduras alográficas, resultantes da tradição manuscrita ou da prática tipográfica, como $<\mathfrak{c h}, \mathfrak{d}, \mathfrak{f f}, \mathfrak{f i}, \mathfrak{f l}, \mathfrak{f t}, \mathfrak{f l}, \mathfrak{f t}, \mathfrak{b}>$ para $<\mathrm{ch}, \mathrm{ck}, \mathrm{ff}, \mathrm{fi}, \mathrm{fl}, \mathrm{ft}, \mathrm{ss}, \mathrm{st}, \mathrm{tz}>$ (veja-se também Killius 1999: 122).

Por outro lado, foi com a data de 3 de janeiro de 1941 (Plata 1999: 68; Beck 2006: 264; Reibold 2010: 10), que a Fraktur (e com ela também a vertente manuscrita da Sütterlinschrift, vinculada à Fraktur desde 1924), teve um fim abrupto e inesperado: uma vez que o governante nacional-socialista Adolf 
Hitler julgou ver na versão Fraktur da chamada 'escrita de Schwabach'9 uma ferramenta dos judeus residentes na Alemanha, mandou divulgar pelo chefe da chancelaria do partido Martin Bormann o chamado 'Normalschrifterlass'. Com esta circular partidária (!), a 'escrita gótica' caiu em descrédito e declarou-se que no futuro a Antiqua deveria ser considerada como 'escrita normal' para a correspondência pública.

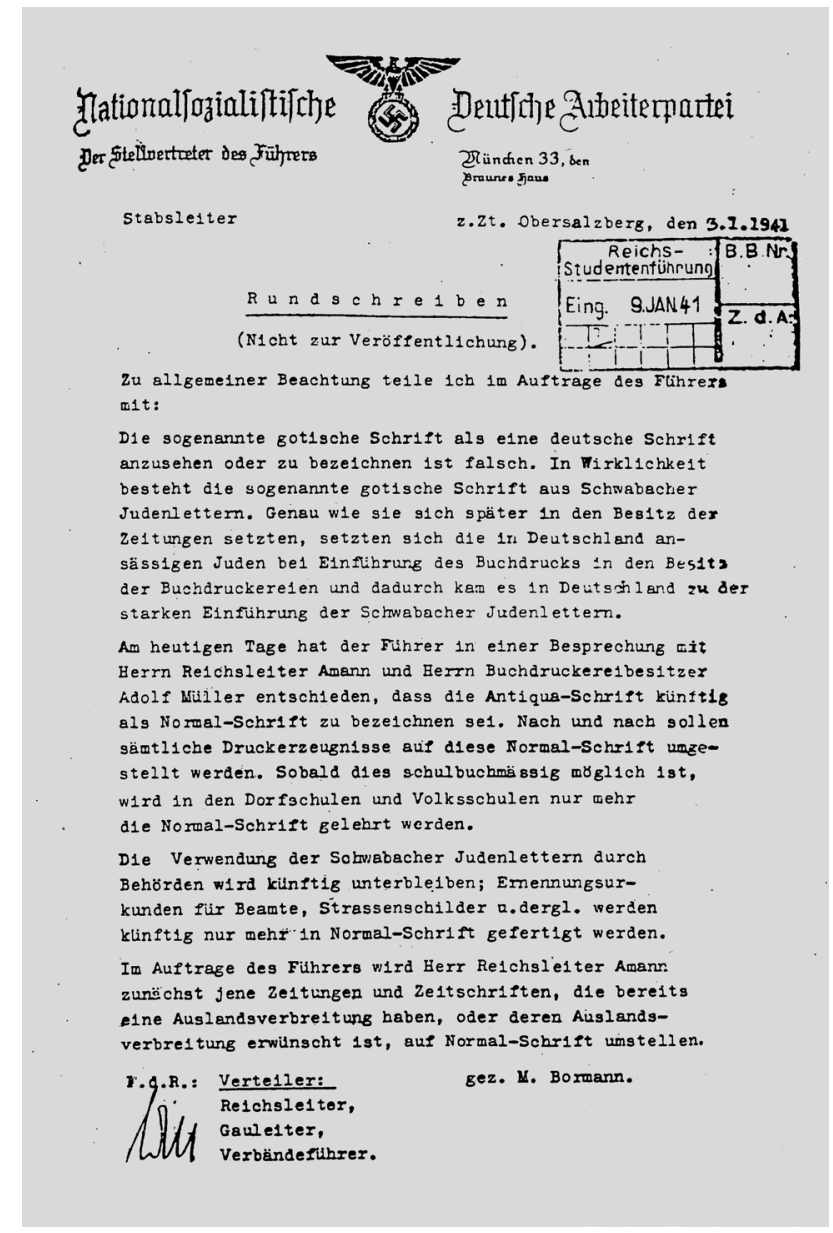

Fac-símile da circular de Martin Bormann (Fonte: Plata 1999: 69). ${ }^{10}$

9 Schwabach é uma cidade do distrito de Mittelfranken, no Estado Federado da Baviera (Bayern) e perto da cidade de Nürnberg. De maneira muito vaga, o gueto dos judeus de Schwabach costuma ser relacionado com a fundição das letras e da impressão de livros em finais do século XV.

10 [Não se traduzem o cabeçalho, a lista de distribuição ou a assinatura: 


\section{Observações finais}

Bernd Marizzi assinala com razão que a biscritualidade ${ }^{11}$ da língua alemã, codificada nas obras metalinguísticas de alemão como língua estrangeira, no período anterior a 1941 exigiram aos estudantes da língua alemã uma enorme esforço adicional, já que estes, para além das peculiaridades da pronúncia e das extensas regras da gramática alemã, também tinham de dominar pelo menos três dos quatro repertórios de carateres impressos e manuscritos supra referidos se eles desejassem obter a capacidade da leitura e da escrita em alemão:

Stehen in unserer heutigen Sicht diese Schwierigkeiten mit Texten der deutschen Sprache vor 1941 unter einer rein historischen Perspektive, so handelte es sich dagegen für Deutschlernende jener Zeit im nichtdeutschsprachigen Ausland um ein gravierendes aktuelles Problem, das den Erfolg ihrer Annäherung an

\footnotetext{
Circular

(Não para publicação)

Para atenção geral, faço o seguinte anúncio por ordem do Führer:

Considerar ou designar a chamada escrita gótica como uma escrita alemã está errado. $\mathrm{Na}$ realidade, a chamada escrita gótica consiste em letras judias de Schwabach. Tal como mais tarde assumiram o propriedade dos jornais, por ocasião da introdução da impressão os judeus residentes na Alemanha assumiram a propriedade das tipografias e, assim, deu-se na Alemanha a maciça introdução das letras judias de Schwabach.

No dia de hoje, o Führer decidiu, numa conversa com o Senhor Reichsleiter Amann e o dono de tipografia Herr Adolf Müller, que a escrita Antiqua no futuro deve ser designada como escrita normal. Todas as publicações devem ser gradualmente convertidas para esta escrita normal. Assim que for viável em termos de manuais didáticos, somente a escrita normal será ensinada nas escolas de aldeia e nas escolas de ensino primário.

O uso das letras judias de Schwabach por entidades oficiais será evitado no futuro; diplomas de nomeação para funcionários públicos, placas de rua etc., no futuro somente serão produzidas em escrita normal.

Por ordem do Führer, O Senhor Reichsleiter Amann converterá em primeiro lugar para a escrita normal]

11 Para além disso, no espaço de língua alemã observava-se ao mesmo tempo a situação de «[...] einer freien, diasituativen Zweischriftigkeit (Biglyphismus), in der aber mehr als $80 \%$ der deutschsprachigen Texte in Fraktur und anderssprachige Texte (oder Textteile) in Antiqua gesetzt werden [...]» [uma coocorrência de duas escritas (biglifismo) livre e diassituativa, em que mais de $80 \%$ dos textos em língua alemã eram compostos em Fraktur e outros textos de outras línguas (ou partes de textos) eram compostos em Antiqua (...)] (Marizzi 2012b: 190), na qual, por exemplo, inserções latinas ou de outras línguas em textos vernaculares alemães eram regularmente distinguidas por meios tipográficos.
} 
die deutsche Sprache und die dazugehörigen Kulturen insofern erschwerte, als die Lernenden sich nicht nur auf linguistischer Ebene ein neues Zeichensystem aneignen mussten, sondern auch neue Zeichensysteme auf graphemischer Ebene, denn im strengen Sinn mussten sie vier Gruppen von neuen Schriftzeichen rezipieren und produzieren. Zu den schon bekannten Groß- und Kleinbuchstaben der Lateinschrift kamen neu hinzu: Groß- und Kleinbuchstaben der deutschen Druckschrift (Fraktur) sowie Groß- und Kleinbuchstaben der deutschen Handschrift (Kurrent/ Sütterlin) (Marizzi 2012b: 188). ${ }^{12}$

Entre os doze manuais de alemão para um público-alvo português que apresentámos e discutimos na nossa recente tese de doutoramento (Kemmler 2018), nenhum dos autores da tradição metalinguística portuguesa prescinde da reprodução de palavras alemãs em Fraktur, havendo inclusive alguns autores que atribuem um papel importante à Kurrentschrift (assim, por exemplo, Apell 1906, I: 10-34). Neste sentido, parece óbvio que a observação de Marizzi, tecida em relação à gramaticografia espanhola de língua alemã, parece semelhantemente verdadeira no tocante aos inícios da tradição portuguesa correspondente:

Es etablierte sich also in Spanien im Laufe des 19. Jahrhunderts eine Konvention der Zweischriftigkeit in spanischen Deutschgrammatiken, in denen der deutsche Text in Fraktur und der spanische in Antiqua gedruckt wurden (Marizzi 2012b: 201)..$^{13}$

Com pequenas alterações, a afirmação supra de Bernd Marizzi pode ser aplicada à situação do assunto deste artigo da seguinte forma: Tal como em Espanha, também em Portugal estabeleceu-se uma convenção de biscritualidade

12 [Se, desde o ponto de vista de hoje, estas dificuldades com os textos da língua alemã anteriores a 1941 não passam de uma perspectiva puramente histórica, para os estudantes de alemã em países de língua não alemã da época constituíam um problema atual sério, que dificultava o sucesso da sua aproximação à língua alemã e às culturas relacionadas no sentido em que os estudantes não apenas tinham que adquirir um novo sistema de signos a nível linguístico, mas também novos sistemas de signos a nível grafémico, pois no sentido estrito tiveram que receber e produzir quatro grupos de carateres. Além das já conhecidas letras maiúsculas e minúsculas da escrita latina, acrescentaram-se: letras maiúsculas e minúsculas da letra de forma (Fraktur), bem como letras maiúsculas e minúsculas da letra manuscrita alemã (Kurrent / Sütterlin)].

13 [Assim, no decorrer do século XIX, estabeleceu-se na Espanha uma convenção de biscritualidade nas gramáticas espanholas da língua alemã, nas quais o texto em alemão era impresso em Fraktur e o espanhol em Antiqua]. 
nos manuais portugueses da língua alemã, nomeadamente no sentido de os respetivo texto alemão ser sempre impresso em Fraktur e o português em Antiqua!

\section{Referências bibliográficas}

APELL, A[lfred]. Nova grammatica theorica e prática para o estudo da lingua allemã. Por A. Apell, Revista por Z. Consiglieri-Pedroso, Lente do curso superior de letras, Paris; Lisboa: Guillard, Aillaud \& C. ${ }^{\text {ia }}$ Casa editora e de commissão, 1897.

APELL, A[lfred]. Nova grammatica theorica e prática para o estudo da

lingua allemã. Por A. Apell, Autor da Grammatica approvada pelo Conselho Superior de Instrucção Publica e adoptada para o $3 .^{\circ}$ anno, Revista por Z. Consiglieri-Pedroso, Lente do curso superior de letras, Paris; Lisboa: Guillard, Aillaud \& C ${ }^{\text {ia }}$ Casa editora e de commissão, 1898a.

APELL, A[lfred]. Selecta de Leituras Allemãs: IV, V, VI Classe dos Lyceus. Paris; Lisboa: Guillard, Aillaud \& C. ${ }^{\text {ia }}$ Casa editora e de commissão (Ensino Secundario Official), 1898b.

APELL, A[lfred]. Grammatica Allemã: primeira parte, por A. Apell, Lente no Curso Superior de Letras, Lisboa: Ferreira \& Oliveira, Limitada, Editores, 1906, I.

BECK, Friedrich. "Schwabacher Judenlettern": Schriftverruf im Dritten Reich. In: Brachmann, Botho / Knüppel, Helmut / Leonhard, Joachim-Felix / Schoeps, Julius H[ans] (Hrsg.). Die Kunst des Vernetzens Festschrift für Wolfgang Hempel. Berlin: Verlag für Berlin-Brandenburg, p. 251-269, 2006. BUNČIĆ, Daniel. Biscriptality in Slavic and non-Slavic languages: a sociolinguistic typology, unveröffentlichte Habilitationsschrift, Tübingen: Universität Tübingen, 2011.

BUNČIĆ, Daniel. History of theoretical research on biscriptality. In: Bunčić, Daniel / Lippert, Sandra L. / Rabus, Achim (eds.). Biscriptality: A sociolinguistic typology. With contributions by Anastasia Antipova, Carmen Brandt, Ekaterina Kislova, Henning Klöter, Alexandra von Lieven, Sandra L. Lippert, Helma Pasch, Achim Rabus, Jürgen Spitzmüller, Constanze Weth, on behalf of the Heidelberg Academy of Sciences and Humanities, the State Academy of Baden-Württemberg, Heidelberg: Universitätsverlag Winter (Akademiekonferenzen; 21), p. 27-50, 2016. 
CAMPOS, A[gostinho] de. Grammatica allemã, por A. de Campos. Professor do Lyceu Central de Lisboa, Paris; Lisboa: Aillaud \& C. ${ }^{\text {ia }}$ Casa Editora e de Commissão (Ensino Secundario Official: Curso Transitorio), 1898a.

CAMPOS, A[gostinho] de. Leituras allemãs. Paris; Lisboa: Aillaud \& C. ia Casa Editora e de Commissão (Ensino Secundario Official: Curso Transitorio), 1898 b.

CORVO SÁNCHEZ, María José. Juan Ángel de Zumaran: un maestro de lenguas español entre los alemanes en el siglo XVII: Una aportación a la historia de la enseñanza de lenguas extranjeras. Salamanca: Universidad de Salamanca, Facultad de Filología, tesis doctoral, 2002.

CORVO SÁNCHEZ, María José. Los libros de lenguas de Juan Ángel de Zumaran: La obra de un maestro e intérprete de lenguas español entre los alemanes del siglo XVII Frankfurt am Main; Berlin; Bern; Bruxelles; New York; Oxford; Wien: Peter Lang (SABEST Saarbrücker Beiträge zur Sprach- und Translationswissenschaft; 12), 2007.

CORVO SÁNCHEZ, María José. Juan Ángel de Zumaran: maestro de lenguas extranjeras en el siglo XVII, Una aportación a la historia de la enseñanza de lenguas extranjeras. Saarbrücken: Editorial Académica Española; Laplambert Academic Publishing, 2011.

GLÜCK, Helmut: Schriften im Kontakt. In: Günther, Hartmut / Ludwig, Otto (Hrsg.). Schrift und Schriftlichkeit: Writing and its Use, Ein interdisziplinäres Handbuch internationaler Forschung, An Interdisciplinary Handbook of International Research, 1. Halbband / Volume 1, Berlin; New York: Walter de Gruyter (Handbücher zur Sprach- und Kommunikationswissenschaften; Handbooks of Linguistics and Communication Science; HSK 10.1), p. 745-766, 1994.

KEMMLER, Rolf. Die Anfänge des Deutschlernens und -lehrens in Portugal: die frühesten Deutschlehrwerke (1863-1926) als Reflex der 'Grammatik-Übersetzungsmethode'?. Vigo: Universidade de Vigo, Escola Internacional de Doutoramento, tese de doutoramento, 2018. Disponível em: http://hdl.handle.net/11093/1093,

KILLIUS, Christina. Die Antiqua-Fraktur-Debatte um 1800 und ihre historische Herleitung. Wiesbaden: Harrassowitz Verlag (Mainzer Studien zur Buchwissenschaft; 7), 1999.

KÜHN, Julius. Gramatica alemana, precedida de un cuadro histórico del origen y progresos de esta lengua, por Don Julio Kühn, Oficial de la Secretaría de la Interpretación de Lenguas, de orden superior, Madrid: En la Imprenta Nacional, 1844. 
MARIZZI, Bernd. La Gramática de la Lengua Alemana de Antonio de Villa (1792): fuentes y correctores. Revista española de lingüistica aplicada 25, p. 133-146, 2012a.

MARIZZI, Bernd. Zweischriftigkeit in frühen Deutschgrammatiken in Spanien. Sprachwissenschaft. 37/2, p. 187-212, 2012 b.

MARTINS, António. Antonij martini primi quondã huius artis pastra ne in alma vniuersitate Ulixbonensi preceptoris, materierũ editio a baculo cecorum breuiter collecta incipit // Jmpressum vero Ulixbone per Ualentinũ ferdinandi de morauia. Anno incarnationis domini Millesimo quadringentesimo nonagesimo septimo. Die vero, xx. mensis junij, 1497.

OLIVEIRA, Fernão de. Grammatica da lingoagem portuguesa. /// Acabouse dempremir esta premeira anotação da lingua Portuguesa. por mandado do muy manifico senhor dom Fernando Dalmada em Lixbõa. ẽ casa d[e] Germão galharde a. xxvij. dias do mes de Janeyro de mil \& q[ui]nhẽtos \& trinta \& seis annos de nossa saluaçam. Deo gratias, 1536.

OVERGAAUW, Eef. Die Nomenklatur der gotischen Schriftarten bei der Katalogisierung von spätmittelalterlichen Handschriften. Codices manuscripti \& impressi 15, p. 100-106, 1994.

PASTRANA, Juan de; ROMBO, Pedro. Grãmatica pastrane /// Jncipit compendium breue et vtile: siue tractatus intitulatus: Thesaurus pauperum siue speculum puerorum editum a magistro Johãne de pastrana. /// [Jmpressa vero Ulixbone per Ualentinũ ferdinandi de morauia. Ad laudẽ õipotentis dei eius ̃̃ genitricis Millesimo quadringentesimo nonagesimo septimo. vj. kalendas Junij],1497.

PLATA, Michael. Die Abschaffung der Deutschen Schrift in schleswig-holsteinischen Tageszeitungen 1941-44. Demokratische Geschichte: Jahrbuch des Beitrats für Geschichte in der Gesellschaft für Politische Bildung Schleswig-Holsteins e.V. 12, p. 61-76, 1999.

REIBOLD, Janina. Verbot der Frakturschriften durch die Nationalsozialisten. un!mut: Zeitschrift an der Uni Heidelberg 206/05 (Themenheft: Nationalsozialismus in Heidelberg, 7. Juli 2010), p. 16, 2010.

ROMBO, Pedro. Materiarum editio ex baculo cecorũ a petro rombo in artibus baccalario breuiter collecta incipit. /// Jmpressa vero Ulixbone per Ualentinũ ferdinandi de morauia. Ad laudẽ omnipotentis dei eiusque genitricis Millesimo quadringentesimo nonagesimo septimo. vj. kalendas Junij,1497.

SCHNEIDER, Karin. Paläographie und Handschriftenkunde für Germanisten: Eine Einführung, 3., durchgesehene Auflage. Berlin; New York: 
De Gruyter (Sammlung kurzer Grammatiken germanischer Dialekte: B, Ergänzungsreihe; 8), 2014.

ŠEDIVÝ, Juraj.: Mittelalterliche Schriftkultur im Pressburger Kollegiatkapitel. Bratislava: Chronos, 2007.

SEIDL, Johannes. Schriftbeispiele des 17. bis 20. Jahrhunderts zur Erlernung der Kurrentschrift: Übungstexte aus Perchtoldsdorfer Archivalien, 2. Auflage, Perchtoldsdorf: Marktgemeinde Perchtoldsdorf (Schriften des Archivs der Marktgemeinde Perchtoldsdorf, 1), 1996.

SÜß, Harald. Deutsche Schreibschrift: Lesen und Schreiben lernen: Lehrbuch, Augsburg: Augustus-Verlag, 1995.

VILLA, Antonio de. Gramatica de la lengua alemana: dividida en tres partes, la primera trata de la forma número, sonido y pronunciacion de las Letras, con las reglas generales de la Prosodia y Ortografia; la segunda procede por todas las partes de la Oracion y la tercera contiene las reglas de la Sintáxis o Contruccion Alemana, con un apendice que contiene por orden alfabetico los generos de los Nombres Substantivos, y concluidos se añade una recopilacion de terminos pertenecientes á diversas facultades; y otra al fin de frases escogidas para empezar a hablar en cosas familiares con la propiedad que corresponde, obra utilisima para la nacion española, Compuesta y arreglada á los preceptos de los dos mejores Maestros Alemanes Matias Kramer, y Juan Christoval Gottsched, Por el padre Lector Fr. Antonio de Villa, del Orden de Predicadores, Cura de Extrangeros de los Reales Hospitales General y Pasion de esta Corte, En Madrid: en la Imprenta Real, 1792.

ZUMARAN, Juan Ángel de. Thesaurvs fvndamentalis qvinqve lingvarvm: hoc est, liber ex qvo velvti clivite cornv copiæ rectissima eaqve facillima methodo, qvidqvid ad pronvntiationem, nominum, iuxta ac verborum inflexionem, Dialogos item \& proverbia, ac tandem ad ipsa quinq[ue] totius Europæ primariarum Linguarum, videlicet Latinæ, Hispanicæ, Gallicæ, Italicæ \& Germanicæ Fundamenta spectare potest, quasi nullo negotio depromitur, \& ad oculum demostratur, Pars prima, In gratiam omnium Natrinum Compositus, Ingolstadtii: Typis Wilhelmi Eder, Svmptibvs Avctoris, 1626.

Articulista convidado 